\title{
Autores más citados en publicaciones periódicas del área de biblioteconomía y documentación: España, 1975-1984
}

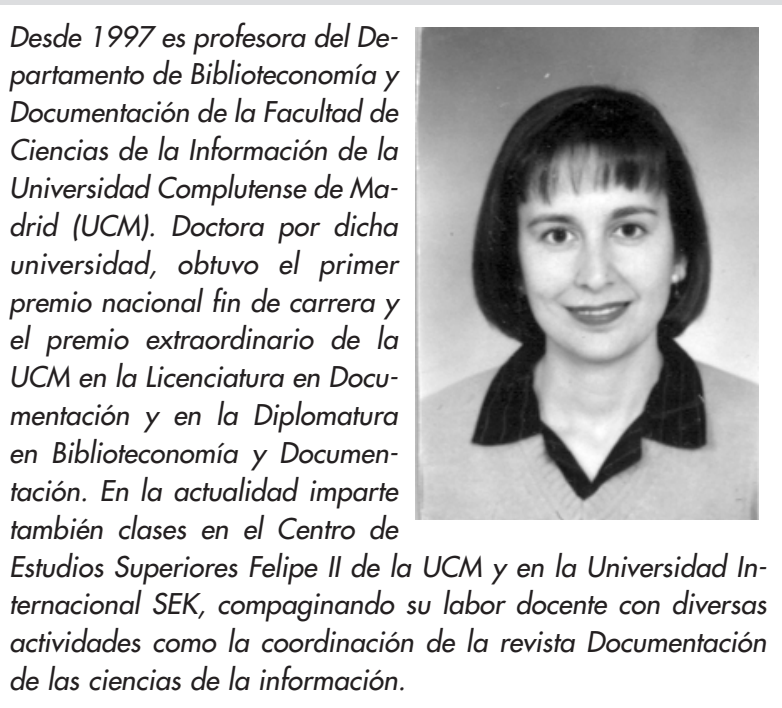

\author{
Por Rosario Arquero Avilés
}

\begin{abstract}
Resumen: En este trabajo se presenta uno de los hallazgos del análisis de citación realizado a partir de un conjunto de publicaciones periódicas del área de biblioteconomía y documentación durante 1975-1984 en España. Concretamente se aporta la relación de autores más citados en dicha etapa en las publicaciones del área que han sido objeto de estudio. Adicionalmente, se establece una comparación con la relación de autores más citados en el período 1985-1998, estudiado por un grupo de investigadores de la Facultad de Biblioteconomía y Documentación de la Universidad de Granada, con el fin de determinar la relación de los autores del área de Biblioteconomía y Documentación más citados desde el año 1975 (dotación de la primera cátedra de Documentación) hasta nuestros días.
\end{abstract}

Palabras clave: Análisis de la investigación española en biblioteconomía y documentación, Análisis de citas, Publicaciones periódicas, Revistas científicas, Actas de congresos.

\section{Title: The most cited authors in library and information science serials: Spain, 1975-1984}

\begin{abstract}
Findings are given of the citation analysis performed on library and information science serials during the period 1975-1984 in Spain. Specifically the most cited authors of these publications are identified. Furthermore, a comparison is offered between these names and those of the most cited authors during the period 1985-1998, which had been studied by researchers at the Faculty of Library and Information Science of the University of Granada. The objective is to provide a list of the most cited authors in Library and Information Science from 1975 (when the first chair in Documentation in Spain was established) to the present day.
\end{abstract}

Keywords: Analysis of Spanish research in library and information science, Citation analysis, Periodical publications, Scientific journals, Proceedings.

Arquero Avilés, Rosario. "Autores más citados en publicaciones periódicas del área de biblioteconomía y documentación: España, 1975-1984”. En: El profesional de la información, v. 11, n. 6, 2002, noviembre-diciembre, pp. 436-441.

\section{Introducción}

En el marco global del análisis realizado sobre la investigación española en biblioteconomía y documentación (análisis de producción, de citación y de cocitación de autores) en el período 1975-1984, se contextualiza el resultado concreto que se presenta en este artículo: un listado de autores más citados en dicho período en una serie de publicaciones periódicas del área que han sido objeto de estudio.

En concreto, dicha relación es un de las consecuencias del análisis de citas llevado a cabo partiendo de los documentos citados en los trabajos-fuente (citantes) aparecidos en un conjunto de publicaciones periódicas del área de biblioteconomía y documentación seleccionadas. Hemos de mencionar la existencia de una serie de trabajos previos en los que se han desa- rrollado estudios de producción, autoría y/o citación de la literatura en biblioteconomía y documentación en España, pero sobre períodos posteriores y/o relaciones de publicaciones del área más reducidas (Frías Montoya; Romero Gómez, 1998), (Jiménez Contreras; Moya Anegón, 1997), (Moya Anegón; Jiménez Contreras, 1999), (López Gijón; Pérez López; Ruiz Villegas del Coso, 1995), (López López, 1996), (Moya Anegón, 2000), (Pérez Álvarez-Ossorio, 1997).

En nuestro caso, y una vez acotado el alcance cronológico del trabajo, hemos de establecer que el análisis realizado sobre la investigación española en este área en general, así como el análisis de citas en particular, se ha basado en una selección de publicaciones periódicas de dicho campo que son, específicamente, revistas científicas y actas de congresos, por haber 
considerado una serie de argumentos que nos han parecido muy razonables:

a. Es necesario emplear el material publicado en revistas y otros medios de comunicación científica (como actas de congresos) para definir el campo de disciplinas muy dinámicas, como es el caso, en donde las fronteras están muy poco claras (Rochester, 1995).

b. La mayor parte de los trabajos que pueden ser tenidos en cuenta como investigación aparecen publicados en revistas (Järvelin; Vakkari, 1990). Así, aunque puede considerarse que la investigación en biblioteconomía y documentación con una orientación más humanística aparece publicada en monografías, tal y como deducen los autores del artículo referenciado, también se trasladan sus resultados a artículos de revista. Por ello, establecen que es posible hacer inferencias fiables sobre la investigación en este campo partiendo de la base de los artículos de revista, sin necesidad de tener que analizar directamente las monografías.

c. El tercero de ellos se resume así: es necesario analizar las comunicaciones a congresos ya que, en disciplinas muy poco consolidadas, tal y como ocurre en nuestro país, los congresos constituyen el principal canal de comunicación para trasladar tanto experiencias como reflexiones y para dar a conocer los incipientes trabajos de investigación (Delgado López-Cózar, 2000).

\section{"Aunque puede considerarse que la investigación en bibliote- conomía con una orientación más humanística aparece pu- blicada en monografías, tam- bién se trasladan sus resulta- dos a artículos de revista»}

En concreto, las publicaciones periódicas (revistas científicas y actas de congreso) con las que se ha trabajado fueron seleccionadas por las siguientes razones:

1. En primer lugar, por aparecer en las notas bibliográficas de una muestra de las monografías escritas en el período 1975-1984 por un conjunto de autores que debían cumplir los siguientes requisitos:

\section{Listado de publicaciones periódicas del área de biblioteconomía y documentación en las que} se ha basado el análisis de citación realizado.

\section{Revistas científicas.}

—Biblioteconomía. Barcelona: Diputació Provincial, Escola de Bibliologia, 1944-1976. Issn 0006-1778.

-Boletín de la Anaba. Madrid: Asociación Nacional de Bibliotecarios, Archiveros y Arqueólogos, 19581977. Issn 0044-9288. Continuada por Boletín de la Anabad.

—Boletín de la Anabad. Madrid, Anabad, 1978- . Issn 0210-4164. Continuación de Boletín de la Anaba.

—-Boletín de la Asociación Andaluza de Bibliotecarios. Málaga, Asociación Andaluza de Bibliotecarios, 1984- . Issn 02।3-6333.

-Boletín de documentación del fondo para la investigación económica y social. Madrid: Fondo para la Investigación Económica y Social de la Confederación Española de Cajas de Ahorros, 1969-1978. Issn 00I5-6I32.

—Boletín Millares Carlo. Las Palmas: Uned, 1980- . Issn 02II-2I40.

—Boletín de la Unesco para las bibliotecas. París: Unesco, 1947-1978. Issn 0304-2979.

—Documentación de las ciencias de la información. Madrid, Universidad Complutense, 1976- . Issn 02104210.

-Revista de archivos, bibliotecas y museos. Madrid: Cuerpo Facultativo de Archiveros, Bibliotecarios y Anticuarios, I87I-1980. Issn 0034-77Ix.

—Revista española de documentación científica. Madrid, Csic, 1977- . Issn 0210-0614.

—Revista de la Unesco de ciencia de la información, bibliotecología y archivología. París: Unesco, 1979- . Issn 0379-12II.

\section{Actas de congresos.}

—Congresos de la Asociación Nacional de Bibliotecarios, Arqueólogos y Documentalistas. 198I- .

-Jornadas españolas de documentación automatizada. 1984- .

-Jornadas bibliotecarias de Andalucía. 198I- . 
- Haber escrito, durante dicha etapa, al menos una monografía cuya materia se adscribiera al área de estudio. Para la obtención de esta información, y con el fin de conseguir una cobertura total, se consultaron 3 fuentes de información: base de datos

\begin{tabular}{|l|c|}
\hline \multicolumn{1}{|c|}{ Autor } & $\mathbf{N}^{\circ}$ de citas recibidas \\
\hline España. Reglamentos. & 88 \\
\hline España. Decretos. & 52 \\
\hline España. Leyes. & 41 \\
\hline España. Cortes Generales. Congreso. & 21 \\
\hline Inspección General de Archivos. & 15 \\
\hline Dirección General de Archivos y Bibliotecas. & 10 \\
\hline Real Academia de la Historia. & 10 \\
\hline España. Cortes Generales. Senado. & 9 \\
\hline Instituto Bibliográfico Hispánico. & 5 \\
\hline Instituto Nacional de Racionalización del Trabajo. & 5 \\
\hline Ministerio de Educación y Ciencia. & 5 \\
\hline
\end{tabular}
Tabla 1. Autores corporativos españoles más citados

Bibliografía General

Española y los catálogos automatizados Ariadna de la Biblioteca Nacional y el de la biblioteca de la Universidad Complutense de Madrid.

- Aparecer en la relación de autores más citados en la fase ya estudiada 1985-1999 (Moya Anegón; Jiménez Contreras, 1999). Hemos de señalar que, de los 43 que aparecen en ella, sólo 22 cumplieron el primero de los requisitos aludido. En concreto, fueron los siguientes: Amat Noguera, Nuria; Caridad Sebastián, Mercedes; Coll-Vinent, Roberto; Currás Puente, Emilia; Desantes Guanter, José María; Escolar Sobrino, Hipólito; Fuentes i Pujol, Ma Eulàlia; García Ejarque, Luis; García Gutiérrez, Antonio Luis; Girón García, Alicia; Heredia Herrera, Antonia; Izquierdo Arroyo, José María; Lasso de la Vega, Javier; López Piñero, José María; López Yepes, José; Mayol i Fernández, Carme; Millares Carlo, Agustín; Moralejo Álvarez, Mª Remedios; Pérez Álvarez-Ossorio, José Ramón; Sagredo Fernández, Félix; Simón Díaz, José y Terrada Ferrandis, María Luz.

\begin{tabular}{|l|c|}
\hline \multicolumn{1}{|c|}{ Autor } & No de citas recibidas \\
\hline Unesco: UN Educational, Scientific and Cultural Organization. & 213 \\
\hline Ifla: Intl. Federation of Library Associations and Institutions. & 44 \\
\hline ONU: Organización de las Naciones Unidas. & 38 \\
\hline FID: Intl. Federation for Information and Documentation. & 24 \\
\hline Unisist: Unesco's World Science Information System. & 19 \\
\hline LoC: Library of Congress. & 18 \\
\hline ISO: International Organization for Standardization. & 16 \\
\hline Ocde: Organización de Cooperación y Desarrollo Económico. & 16 \\
\hline ISI: Institute for Scientific Information. & 13 \\
\hline FAO: Food and Agriculture Organization of the United Nations. & 11 \\
\hline Comisión de las Comunidades Europeas. & 10 \\
\hline Library Association. & 10 \\
\hline British Library. & 7 \\
\hline Centro de Bibliotecología y Metodología Bibliotecológica. & 6 \\
\hline Gobierno de los EUA. & 6 \\
\hline Papua New Guinea University of Technology. & 6 \\
\hline Academia de Ciencias de la Urss. & 5 \\
\hline American Library Association (ALA). & 5 \\
\hline Consejo de Europa. & 5 \\
\hline National Academy of Sciences. & 5 \\
\hline National Library of Australia. & 5 \\
\hline
\end{tabular}

Tabla 2. Autores corporativos extranjeros más citados
Hemos de poner de manifiesto que la consideración de los dos criterios descritos justifica la presencia de las dos publicaciones de Unesco tenidas en cuenta como material-base. Además, a posteriori, al llevar a cabo el cómputo de citas por publicaciones que constituyeron el material-base de la investigación, resultó que ambas, agrupadas bajo el título facticio UnescoBoletín Bibliotecas, por ser una continuación de la otra (aun siendo las únicas publicaciones-base no editadas en España), fueron las que mayor número de citas habían recibido en el período estudiado.

2. En segundo lugar, por cumplir una serie de condiciones como publicaciones de carácter científico según las pautas aportadas por las normas UNE 50-13394 (Documentación. Presentación de artículos en publicaciones periódicas y en serie) y UNE 50-101-90 (Documentación. Presentación de las publicaciones periódicas). Así pues, hemos de señalar que las relaciones de autores más citados que se presentan se refieren exclusivamente a las revistas seleccionadas que son presentadas en el listado correspondiente adjunto a este artículo.

\section{Distribución de citas por autores $y$ autores más citados en el período 1975 - 1984}

Antes de analizar las tablas que se presentarán en este apartado, es preciso hacer una serie de aclaraciones sobre la información que contienen. Se muestran las de los autores corporativos y personales (extranjeros y españoles) que han reci- 
bido 5 citas o más en los trabajos-fuente de las publicaciones seleccionadas, figurando el cómputo total de ellas asociadas a cada uno. Además, se presenta la tabla completa de los autores personales españoles que han recibido alguna cita en dichos documentos.

Ha de tenerse en cuenta que las relaciones de investigadores que se presentan se derivan de la información obtenida a partir de las publicaciones periódicas que constituyen el material-base en el que se sustenta el trabajo.

\section{«En general puede establecer- se que predominan los autores personales de trabajos citados frente a los corporativos»}

En la presentación de la información por tablas diferenciaremos por tanto entre autores personales españoles y extranjeros y autores corporativos españoles y extranjeros (ver tablas 1, 2, 3 y 4). De un análisis global de ellas pueden derivarse una serie de ideas que exponemos a continuación.

En general puede establecerse que predominan los autores personales de trabajos citados frente a los corporativos. De la tabla de los corporativos españoles más citados puede deducirse que muchas de las citas corresponden a trabajos de carácter legislativo y jurídico. Dicha circunstancia se debe a que la mayor parte de los trabajos-fuente aparecidos en las publicaciones periódicas seleccionadas tratan aspectos de carácter general, teórico e histórico, tratándose de materiales en los que se citan frecuentemente fuentes legislativas y jurídicas.

Destaca la aparición de la Unesco como autor corporativo más citado, consecuencia de la presencia de las dos publicaciones periódicas editadas por dicha institución: Boletín de la Unesco para las bibliotecas y Revista de la Unesco de ciencia de la información, bibliotecología y archivología, que fueron tenidas en cuenta como material-base en función de los criterios explicados anteriormente y aplicados en la selección realizada. En este sentido, no podemos obviar el papel de los trabajos editados por esta organización en el marco del programa Unisist y su influencia, en el período cronológico analizado, en países como España en los que nuestra disciplina se encontraba en un estado embrionario.

Obsérvese que las dos primeras posiciones de la tabla de autores personales más citados las ocupan Line y Garfield, ambos adscritos a la especialidad bibliometría y/o informetría, y, por ello, muy citados por personas que habitualmente publican trabajos de ca-

\begin{tabular}{|c|c|}
\hline Autor & $\begin{array}{c}\mathrm{N}^{\circ} \mathrm{de} \\
\text { citas } \\
\text { recibidas }\end{array}$ \\
\hline Line, Maurice $B$. & 29 \\
\hline Garfield, Eugene. & 24 \\
\hline Sandoval, Armando M. & 18 \\
\hline Price, Derek J. de Solla. & 17 \\
\hline Brookes, Bertram C. & 15 \\
\hline Shera, Jesse Hauk. & 14 \\
\hline Vickery, Brian C. & 14 \\
\hline Bradford, Samuel Clement. & 13 \\
\hline Anderson, Dorothy. & 12 \\
\hline Büttenklepper, A. & 12 \\
\hline Mijailov, O. A. & 12 \\
\hline Delmas, Bruno. & 11 \\
\hline Gylyarevsky, R. S. & 10 \\
\hline Viet, Jean. & 10 \\
\hline Evans, A. J. & 9 \\
\hline Grolier, Eric de. & 9 \\
\hline Bousso, Amadou. & 8 \\
\hline Kent, Allen. & 8 \\
\hline Lancaster, F. Wilfrid. & 8 \\
\hline Martyn, John. & 8 \\
\hline Saracevic, Tefko. & 8 \\
\hline Almada de Ascensio, Margarita. & 7 \\
\hline Anderla, Georges. & 7 \\
\hline Atherton, Pauline. & 7 \\
\hline Chaumier, Jacques. & 7 \\
\hline Chernyi, A. I. & 7 \\
\hline Duchein, Michel. & 7 \\
\hline Foskett, Douglas John. & 7 \\
\hline Neelameghan, $\mathrm{A}$. & 7 \\
\hline Otlet, Paul. & 7 \\
\hline Roper, Michael. & 7 \\
\hline Wersig, Gernot. & 7 \\
\hline Wilson, T. D. & 7 \\
\hline Bell, I. & 6 \\
\hline Freeman, Robert R. & 6 \\
\hline Griffiths, J. M. & 6 \\
\hline Gross, E. M. & 6 \\
\hline Gross, P. L. K. & 6 \\
\hline Penna, Carlos Víctor. & 6 \\
\hline Pérez-Guinjoán, A. & 6 \\
\hline Poncelet, J. & 6 \\
\hline Rigby, Malcom. & 6 \\
\hline Wynar, Lubomyr, R. & 6 \\
\hline Apor-Szeidovitz, Eva. & 5 \\
\hline Asís, Moisés. & 5 \\
\hline Avram, Henriette D. & 5 \\
\hline Borko, Harold. & 5 \\
\hline
\end{tabular}

Tabla 3. Autores personales extranjeros más citados 
rácter bibliométrico en la $R e$ vista española de documentación científica y cuya procedencia institucional es el Consejo Superior de Investigaciones Científicas.

\section{Comparativa entre las relaciones de autores personales españoles más citados en los períodos 1975-1984 y 1985-1998}

Nos parece especialmente interesante completar el análisis de la tabla de autores personales nacionales presentada en el anterior apartado con la llevada a cabo por el equipo de la Universidad de Granada y que puede verse en la tabla 5 (Moya Anegón; Jiménez Contreras, 1999).

La comparación entre ambas nos permite extraer las siguientes conclusiones:

Solamente encontramos un autor que ocupa la misma posición en ambos períodos. Adicionalmente hemos de decir que se trata de la primera posición, desempeñada en las dos tablas por el profesor José López Yepes. Este hecho permite establecer que aparece como referente de la biblioteconomía y documentación españolas de los últimos 25 años. Pensamos que dicha circunstancia puede deberse a que se trata del primer catedrático de documentación de nuestro país.

\section{«José López Yepes aparece co- mo referente de la biblioteco- nomía y documentación espa- ñolas de los últimos 25 años»}

En el resto de los casos se han producido variaciones de posición entre uno y otro período, de modo que podemos observar la siguiente situación:

-Autores que han pasado a ocupar una mejor posición en el período más reciente y que por tanto se han

\begin{tabular}{|c|c|c|c|}
\hline Autor & Citas & Autocitas & C-A \\
\hline López Yepes, José. & 35 & 8 & 27 \\
\hline Lasso de la Vega, Javier. & 21 & 0 & 21 \\
\hline Millares Carlo, Agustín. & 17 & 1 & 16 \\
\hline Méndez Miaja, Aida. & 18 & 3 & 15 \\
\hline Terrada Ferrandis, María Luz. & 18 & 4 & 14 \\
\hline Cortés Alonso, Vicenta. & 18 & 6 & 12 \\
\hline Desantes Guanter, José María. & 35 & 25 & 10 \\
\hline Viesca Espinosa, Rosa de la. & 13 & 3 & 10 \\
\hline Sagredo Fernández, Félix. & 11 & 2 & 9 \\
\hline Mateu y Llopis, Felipe. & 9 & 1 & 8 \\
\hline Heredia Herrera, Antonia. & 7 & 0 & 7 \\
\hline Gallego Domínguez, Olga. & 12 & 5 & 7 \\
\hline López Gómez, Pedro. & 14 & 7 & 7 \\
\hline López Piñero, José María. & 16 & 9 & 7 \\
\hline Pérez Álvarez-Ossorio, J. R. & 12 & 5 & 7 \\
\hline Pescador del Hoyo, Ma Carmen. & 8 & 1 & 7 \\
\hline Ferreiro Aláez, Luis. & 14 & 8 & 6 \\
\hline Currás, Emilia. & 8 & 4 & 4 \\
\hline Sancho Lozano, Rosa. & 4 & 1 & 3 \\
\hline Lara Guitard, Alfredo. & 11 & 8 & 3 \\
\hline Navarro, Víctor. & 3 & 1 & 2 \\
\hline Mateu Ibars, Josefina. & 4 & 2 & 2 \\
\hline Bohigas, Pedro & 2 & 1 & 1 \\
\hline García Sevilla, Lluis. & 2 & 1 & 1 \\
\hline Pérez-Rioja, José Antonio. & 2 & 1 & 1 \\
\hline García Gutiérrez, Antonio Luis. & 5 & 4 & 1 \\
\hline López Yepes, Alfonso. & 4 & 3 & 1 \\
\hline Vidal, Xavier. & 2 & 1 & 1 \\
\hline Carrión Gútiez, Manuel. & 1 & 0 & 1 \\
\hline Casabán Moya, Enric. & 1 & 1 & 0 \\
\hline Contreras de Miquel, Remedios. & 3 & 3 & 0 \\
\hline Cabrillana Ciézar, Nicolás. & 5 & 5 & 0 \\
\hline Valle bracero, Antonio. & 5 & 5 & 0 \\
\hline Fernández García, Justo A. & 2 & 2 & 0 \\
\hline Rivas Palá, María. & 2 & 2 & 0 \\
\hline García Melero, Luis Ángel. & 1 & 1 & 0 \\
\hline Mestre, Anna. & 1 & 1 & 0 \\
\hline Peris, Rafael. & 1 & 1 & 0 \\
\hline Riesco Terrero, Ángel. & 1 & 1 & 0 \\
\hline Rodríguez de Diego, José Luis. & 1 & 1 & 0 \\
\hline Torres Escamez, María Del Mar. & 1 & 1 & 0 \\
\hline Barrionuevo Gil, María Francisca. & 1 & 1 & 0 \\
\hline Romero de Tejada, Pilar. & 1 & 1 & 0 \\
\hline Rodríquez Delgado, Rafael. & 12 & 12 & 0 \\
\hline Olaechea Labayen, Juan B. & 2 & 2 & 0 \\
\hline Osuna Ruiz, Manuel. & 1 & 1 & 0 \\
\hline Pereda Alonso, Araceli. & 1 & 1 & 0 \\
\hline Higueruela, Leandro. & 1 & 1 & 0 \\
\hline
\end{tabular}

Tabla 4. Autores personales españoles más citados (período 1975-1984)

consolidado en el área. Es el caso de José María López Piñero, Félix Sagredo Fernández, Manuel Carrión Gútiez y Antonio García Gutiérrez.

- También encontramos el caso contrario, es decir, se advierte que determinados autores ocupan posiciones más bajas en el período más cercano. Quizás los casos más destacables sean los de Javier Lasso de la Vega y Agustín Millares Carlo. La explicación la encontramos si tenemos en cuenta que ambos fallecieron en los años siguientes.

-Otro grupo sería el de aquellos cuya situación podríamos calificar como "equilibrada", pues ocupan posiciones muy similares en ambos períodos. Es el caso de María Luz Terrada Ferrandis, José Ramón 
Pérez Álvarez-Ossorio y Emilia Currás, que aparecen como claros referentes en ambos casos.

Finalmente podemos establecer que quienes aparecen en la relación de 1975-1984, y que muestran su permanencia en la correspondiente de los años más recientes, podrían considerarse "autores clásicos" de la biblioteconomía y documentación de los últimos 25 años. Se trata concretamente de: Carrión Gútiez, Manuel; Currás Puente, Emilia; Desantes Guanter, José María; Ferreiro Aláez, Luis; García Gutiérrez, Antonio Luis; Heredia Herrera, Antonia; Lasso de la Vega, Javier; López Piñero, José María; López Yepes, José; Méndez Miaja, Aida; Millares Carlo,
Agustín; Pérez Álvarez-Ossorio, José Ramón; Sagredo Fernández, Félix y Terrada Ferrandis, María Luz.

\section{Bibliografía}

Delgado López-Cózar, E. «Diagnóstico de la investigación en biblioteconomía y documentación en España (1976-1996): estado embrionario». En: Revista de investigación iberoamericana en ciencia de la información y documentación, 2000, v. 1, n. 1, pp. 79-93.

Frías, J. A.; Romero Gómez, P. «¿Quiénes son y qué citan los investigadores que publican en las revistas españolas de biblioteconomía y documentación?». En: Anales de documentación, 1998, n. 1, pp. 29-53.

Järvelin, K.; Vakkari, P. «Content analisys of research articles in library and information science». En: Library and information science research, 1990, n. 12 , pp. 395-421.

\begin{tabular}{|l|c|c|c|}
\hline \multicolumn{1}{|c|}{ Autor } & Citas & Autocitas & C-A \\
\hline López Yepes, José. & 170 & 7 & 163 \\
\hline Amat Noquera, Núria. & 79 & 1 & 78 \\
\hline López Piñero, José María. & 74 & 0 & 74 \\
\hline Saaredo Fernández, Félix. & 69 & 2 & 67 \\
\hline Anqlada i de Ferrer, Lluís Ma. & 70 & 6 & 64 \\
\hline Carrión Gútiez, Manuel & 64 & 0 & 64 \\
\hline Terrada Ferrandis, María Luz. & 77 & 13 & 64 \\
\hline Pérez Álvarez-Ossorio, José Ramón. & 86 & 25 & 61 \\
\hline Codina Bonilla, Lluís. & 72 & 17 & 55 \\
\hline García Gutiérrez, Antonio Luis. & 60 & 5 & 55 \\
\hline Méndez Miaja, Aida. & 65 & 10 & 55 \\
\hline Coll-Vinent, Roberto. & 52 & 0 & 52 \\
\hline Moscoso Castro, Purificación. & 53 & 2 & 51 \\
\hline Currás Puente, Emilia. & 140 & 90 & 50 \\
\hline Fuentes i Puiol, Ma Eulàlia. & 63 & 13 & 50 \\
\hline Caridad Sebastián, Mercedes. & 49 & 0 & 49 \\
\hline Mavol i Fernández, Carme. & 55 & 6 & 49 \\
\hline Desantes Guanter, José María. & 59 & 11 & 48 \\
\hline Estivill i Rius, Assumpció. & 52 & 4 & 48 \\
\hline Izquierdo Arroyo, José María. & 55 & 9 & 46 \\
\hline Moraleio Álvarez, Ma Remedios. & 46 & 0 & 46 \\
\hline Escolar Sobrino, Hibólito. & 40 & 0 & 40 \\
\hline Pinto Molina, María. & 45 & 5 & 40 \\
\hline Gómez Caridad, Má Isabel. & 52 & 14 & 38 \\
\hline Aqenio Bullón, Xavier. & 90 & 54 & 36 \\
\hline Girón García, Alicia. & 35 & 0 & 35 \\
\hline Moya Aneqón, Félix de. & 41 & 6 & 35 \\
\hline Cornella, Alfons. & 35 & 1 & 34 \\
\hline García Melero, Luis Ánael. & 40 & 6 & 34 \\
\hline Vázauez Valero, Manuela. & 53 & 19 & 34 \\
\hline Lasso de la Veqa, Javier. & 32 & 0 & 32 \\
\hline Abadal Falqueras, Ernest. & 38 & 7 & 31 \\
\hline Massísimo i Sánchez de Boado, Ánqels. & 34 & 3 & 31 \\
\hline García Eiaraue, Luis. & 35 & 5 & 30 \\
\hline Ríos García, Yolanda. & 33 & 3 & 30 \\
\hline Heredia Herrera, Antonia. & 28 & 0 & 28 \\
\hline Simón Díaz, José. & 28 & 0 & 28 \\
\hline Hípola, Pedro. & 30 & 3 & 27 \\
\hline Moreiro González, José Antonio. & 27 & 0 & 27 \\
\hline Sanz Casado, Elías. & 36 & 9 & 27 \\
\hline Millares Carlo, Aqustín. & 26 & 0 & 26 \\
\hline Pulido, Marta. & 32 & 0 & 26 \\
\hline Ferreiro Aláez, Luis. & 7 & 25 \\
\hline
\end{tabular}

Tabla 5. Autores personales españoles más citados (período 1985-1998)
Jiménez Contreras, E.; Moya Anegón, F. «Análisis de la autoría en revistas españolas de biblioteconomía y documentación, 1975-1995». En: Revista española de documentación científica, 1997, v. 20, n. 3, pp. 252-267.

López Gijón, J.; Pérez López, A.; Ruiz de Villegas del Coso, M. «Siete jornadas bibliotecarias de Andalucía». En: VIII Jornadas bibliotecarias de Andalucía, 1995.

López López, P. «La investigación bibliométrica en España (tesis doctorales)». En: Revista española de documentación científica, 1996, v. 19, n. 1, pp. 84-89.

Moya Anegón, F. «La investigación española en recuperación de información (Ri): análisis bibliométrico (19841999)». En: Revista de investigación iberoamericana en ciencia de la información y documentación, 2000, v. 1, n. 1, pp. 117-123.

Moya Anegón, F.; Jiménez Contreras, E. «Autores españoles más citados en biblioteconomía y documentación». En: El profesional de la información, 1999. v. 8, n. 5, pp. 28-29.

Pérez Álvarez-Ossorio, J. R. «Cobertura temática y procedencia institucional de los artículos publicados en la Revista española de documentación científica en sus veinte años de existencia». En: Revista española de documentación científica, 1997, v. 20, n. 4, pp. 290-298.

Rochester, M. K. «Library and information science research in Australia 19851994: a content analysis of research articles in the Australian library journal and Australian Academic \& Research Libraries». En: Australian Academic \& Research Libraries, 1995, n. 26, pp. 163-170.

Rosario Arquero Avilés, Departamento de Biblioteconomía y Documentación de la Facultad de Ciencias de la Información de la Universidad Complutense de Madrid. carquero@ccinf.ucm.es rarquero@cesfelipesegundo.com 